\title{
REVIEWS
}

\section{Ecology and genomics of Actinobacteria: new concepts for natural product discovery}

\author{
Doris A. van Bergeijk (10), Barbara R. Terlouw ${ }^{2,4}$, Marnix H. Medema $\mathbb{1}^{2 凶}$ and \\ Gilles P. van Wezel $\mathbb{D}^{1,3 凶}$
}

Abstract | Actinobacteria constitute a highly diverse bacterial phylum with an unrivalled metabolic versatility. They produce most of the clinically used antibiotics and a plethora of other natural products with medical or agricultural applications. Modern 'omics'-based technologies have revealed that the genomic potential of Actinobacteria greatly outmatches the known chemical space. In this Review, we argue that combining insights into actinobacterial ecology with state-of-the-art computational approaches holds great promise to unlock this unexplored reservoir of actinobacterial metabolism. This enables the identification of small molecules and other stimuli that elicit the induction of poorly expressed biosynthetic gene clusters, which should help reinvigorate screening efforts for their precious bioactive natural products.

Siderophores

Chelating specialized metabolites produced by bacteria and fungi, which form a soluble complex with iron and thereby facilitate the uptake of this essential element.
'Microbial Biotechnology, Institute of Biology, Leiden University, Leiden, The Netherlands.

2Bioinformatics Group, Wageningen University Wageningen, The Netherlands.

${ }^{3}$ Department of Microbial Ecology, Netherlands Institute of Ecology, Wageningen, The Netherlands.

${ }^{4}$ These authors contributed equally: Doris A. van Bergeijk, Barbara R. Terlouw

凶e-mail:marnix.medema@ wur.nl;g.wezel@ biology.leidenuniv.nl https://doi.org/10.1038 s41579-020-0379-y
The phylum Actinobacteria represents one of the most diverse groups of microorganisms in nature. These Gram-positive bacteria have a high GC content and show a remarkable range of morphologies, including unicellular cocci or rods (for example, members of the genera Micrococcus and Mycobacterium), and morphologically complex multicellular bacteria (for example, members of the genera Amycolatopsis, Frankia and Streptomyces $)^{1}$. Actinobacteria are widely distributed across both terrestrial and aquatic ecosystems, as well as in the microbiomes of higher eukaryotes ${ }^{2}$. This ecological diversity is reflected in their metabolic potential as Actinobacteria are extremely versatile producers of bioactive natural products. Notably, Actinobacteria produce two thirds of all known antibiotics used in the clinic today, but also a vast array of anticancer compounds, immunosuppressants, anthelmintics, herbicides and antiviral compounds, in addition to extracellular enzymes ${ }^{3-6}$. Therefore, these bacteria are attractive sources for clinical drugs ${ }^{4,7}$.

The introduction of antibiotics in the twentieth century greatly contributed to the extension of the human lifespan and has saved millions of lives worldwide. However, with the increase in antibiotic resistance, we now face a huge challenge in treating infections by multidrug-resistant bacteria ${ }^{8}$. This coincides with a dramatic decrease in the success of traditional drug development through high-throughput screening ${ }^{9,10}$. Indeed, the chance of finding new antibiotics via traditional methods in randomly chosen Actinobacteria has been estimated at less than one per million ${ }^{11,12}$. However, advances in genome sequencing have unveiled a vast reservoir of biosynthetic gene clusters (BGCs) for natural products in microbial genomes, even in those that had been studied extensively for decades ${ }^{13-15}$. The OSMAC strategy (one strain many compounds) ${ }^{16}$ for extensive mining of individual strains still yields promising new molecules ${ }^{17,18}$. However, the rate of success has decreased dramatically since the golden years of drug discovery, primarily due to replication ${ }^{12}$. The apparent failure to uncover the full potential of natural product-producing microorganisms is likely due to the fact that we lack the understanding that is required to activate the expression of their BGCs in the laboratory. During industrial screening, bacteria and fungi are typically grown in isolation with ample nutrients and resources, which is in sharp contrast to their natural complex and rapidly changing habitat. Antibiotics are believed to be important for survival as mediators of resource competition in a competitive environment ${ }^{19,20}$, and microbial interactions have a key role in their activation ${ }^{21-23}$. Hence, to increase the success of natural product-based drug discovery, we need to elucidate the triggers and cues that activate the expression of BGCs ${ }^{24,25}$.

Bioactive metabolites mediate important ecological functions, which are as diverse as their chemical structures. Siderophores enhance iron uptake in environments where the bioavailability of iron is limited ${ }^{26}$, pigments provide protection against ultraviolet radiation and have antioxidant activity ${ }^{27}$ and compatible solutes protect against osmotic stress ${ }^{28}$. However, the most obvious ecological purpose is biological weaponry to outcompete 
Endospore

A dormant, non-reproductive structure that can survive for long periods of time and is important for dispersal of the organism in the environment.

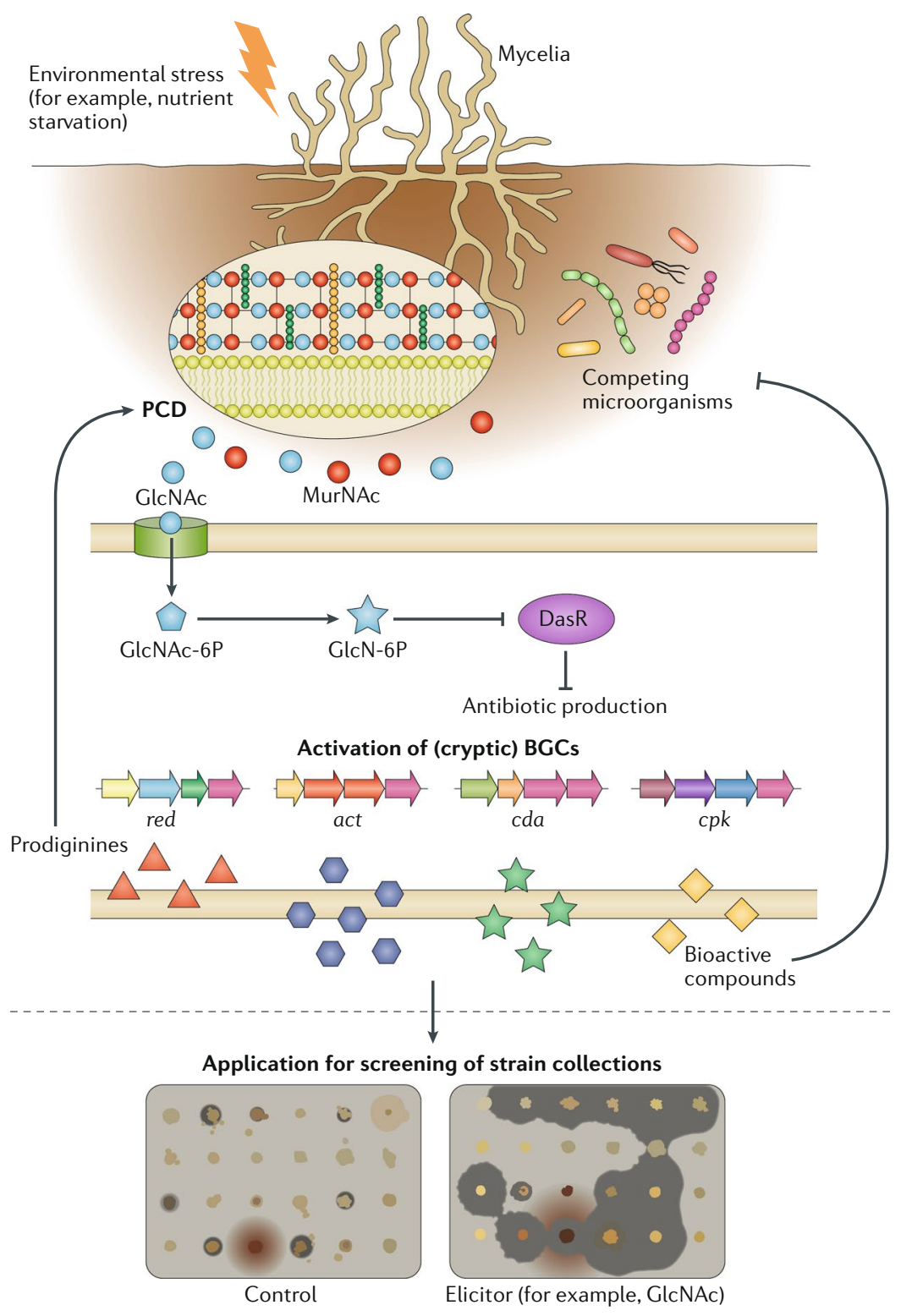

Fig. 1 | From biological understanding to elicitation of BGCs. Upon environmental stress, such as nutrient starvation, programmed cell death (PCD) leads to the autolytic degradation of the old mycelia, which liberates the necessary nutrients to fuel the onset of development and the new biomass. The onset of morphological development correlates temporally with the production of antibiotics. Specifically, cell-wall peptidoglycan is recycled to release the amino sugars $\mathrm{N}$-acetylmuramic acid (MurNAc) and $\mathrm{N}$-acetylglucosamine (GlcNAc). GlcNAc is internalized as GlcNAc-6P and converted to GlcN-6P, which functions as a ligand for the global regulator DasR. Binding of GlcN-6P inactivates the repressor DasR, which results in the derepression of pathway-specific activators of antibiotic biosynthetic gene clusters (BGCs) ${ }^{49}$. The resulting production of antibiotics (such as calcium-dependent antibiotic (Cda), coelimycin P1 (Cpk) and actinorhodin (Act)) is likely to provide a line of defence to protect the nutrients that have been released by PCD against motile saprophytic bacteria, whereas the synthesis of DNA-degrading prodiginines (undecylprodigiosin (Red)) promotes PCD. GlcNAc elicits the transcription of BGCs that are not expressed under standard laboratory conditions and can thus be used in screening regimes for drug discovery. wasp larvae from various pathogenic fungi and bacteria $^{29}$, and endophytic Actinobacteria protect their host against phytopathogens ${ }^{2,30}$.

In this Review, we discuss why Actinobacteria excel as natural product producers and how their specialized metabolism and the regulatory mechanisms governing this metabolism have evolved in the context of ecology and genomic structure. Finally, we explore how ecological insights can be translated into approaches for computational and experimental genome mining strategies that yield novel bioactive molecules, in particular antibiotics.

\section{A mycelial lifestyle}

The propensity to produce bioactive molecules and the richness of bacterial genomes in terms of BGC diversity have been correlated with key organismal features, such as multicellularity, endospore formation and genome size $^{31,32}$. Bacteria can be divided broadly into two groups based on their adaptability: specialists and generalists, each with their own environmental niches. Specialists are dedicated to life in specific environments and therefore require less extensive metabolism and, accordingly, smaller genomes ${ }^{33,34}$. Mycoplasma genitalium is a well-known example of an organism with a small genome of around $580 \mathrm{~kb}$ and fewer than 500 genes $^{35}$. The smallest genomes known to date belong to parasites and symbionts, with the beetle symbiont Stammera spp. as a remarkable example of an organism with a small genome of around $270 \mathrm{~kb}$ and 250 genes $^{36}$.

By contrast, generalists usually have larger genomes and a complex morphology, such as multicellularity and the formation of endospores ${ }^{33,34}$. Their ability to use multiple nutrient sources enables them to adapt to diverse environments and growth conditions, which requires complex metabolic regulation. Therefore, it is not surprising that this group of bacteria includes the most important producers of natural products such as Actinobacteria, Cyanobacteria and Myxobacteria ${ }^{34,37}$. Hallmark features of multicellularity and development include intraspecies communication, morphological differentiation and programmed cell death (PCD) $)^{38}$.

The linkage between morphological and chemical differentiation is best explained using Streptomyces as an example. Streptomycetes are mycelial organisms that reproduce by sporulation, with a life cycle similar to that of filamentous fungi. When the conditions are favourable, a single and uninucleoid spore will germinate and the hyphae grow out by a combination of apical growth and branching, which results in a complex mycelial network ${ }^{3,39}$. Exo-enzymes are released to break down natural polymers, such as cellulose, mannan and chitin, thereby providing nutrients. The vegetative hyphae are compartmentalized by occasional semi-permeable cross-walls to form large multinucleoid cells. The next step in the developmental programme is the formation of new sporogenic aerial hyphae, which eventually differentiate into chains of uninucleoid spores ${ }^{3,39}$. To fuel the onset of the developmental programme, old vegetative mycelia are autolytically degraded through PCD to liberate the necessary nutrients for the new biomass ${ }^{40}$ (FIG. 1). Eventually, reproductive aerial hyphae differentiate into long chains of spores. The onset of development 


\section{Programmed cell death \\ (PCD). A cellular suicide \\ mechanism found in \\ eukaryotes and prokaryotes \\ that is activated in response to \\ different stimuli, including \\ biotic and abiotic stresses. \\ Vegetative hyphae \\ Long filamentous cells with \\ multiple chromosomes that \\ grow by tip extension and \\ branching, forming a dense \\ network into the surrounding \\ substrate (often referred to as \\ the vegetative or substrate \\ mycelium).}

Sporogenic aerial hyphae

Reproductive hyphal structures that emerge from the

vegetative mycelium into the

air and develop into chains of

unigenomic spores.

Saprophytic bacteria

Bacteria, often found in the

soil, that feed on dead and

decaying organic material. is controlled by the bld (bald) genes, so-called because mutants fail to produce the fluffy aerial mycelium ${ }^{41}$. Genes that control the distinct steps leading towards the maturation of aerial hyphae and subsequent sporulation are called whi (white) genes, referring to the white appearance of mutants due to their failure to produce grey-pigmented spores ${ }^{42}$.

The onset of morphological development correlates temporally with the production of antibiotics ${ }^{43,44}$. The production of antibiotics is likely to provide a line of defence to protect the PCD-released nutrients against motile saprophytic bacteria, while at the same time, antibiotic-mediated lysis of these competitors may serve as an alternative food source $e^{3,38,45,46}$. Interestingly, prodiginines - which have DNA-damaging properties and are not secreted, and hence damage the DNA of the producer - apparently facilitate PCD in Streptomyces coelicolor $^{47}$. This suggests that some antibiotics may even drive development via the initiation of PCD.

\section{Missing signals and cryptic antibiotics}

The genome sequences of extant Actinobacteria 'document' their present and (recent) past ecology. They specify various functionalities - including the natural products they produce - used to cope with challenges that these bacteria face in their specific niches. Not surprisingly, genome sequencing has had a huge impact on natural product discovery. This is exemplified by

\section{Box 1 | Silent biosynthetic gene clusters}

What we have learned from genome sequencing efforts it is that many of the biosynthetic gene clusters (BGCs) are not accounted for in the corresponding metabolomes. These BGCs are referred to as 'silent' or 'cryptic'. Although these terms are sometimes interchanged, they have different meanings. A BGC is 'cryptic' when it has been identified but cannot yet be linked to a product, activity or phenotype. To establish whether a cluster is 'silent', experimental validation is required, at the level of gene expression or metabolomics (in case the compound is known). Silence of a BGC may reflect the fact that we do not yet understand the environmental conditions that are required for their expression. Alternatively, a BGC may have been silenced as a first step towards loss of the complete cluster. A transcriptome study of Salinispora strains revealed that more than half of their BGCs were expressed at levels that should facilitate discovery of the compounds they produce ${ }^{156}$. As transcriptome data of many BGCs are lacking, this suggests that most of the unexplored BGCs may be cryptic - that is, not linked to their products - rather than silent. For these BGCs, our failure to detect the corresponding metabolites is linked to other factors, such as translation efficiency and extraction methods.

Although the importance of 'omics'-based natural product discovery is clear ${ }^{157}$, a proportion of BGCs remain transcriptionally silent in the laboratory. Transcriptome analysis revealed that biosynthetic potential is more complicated than the presence or absence of a BGC alone, as a substantial proportion of the BGCs were differentially expressed between strains ${ }^{156}$. An argument often heard during discussions between scientists in the field is that if a few different isolates of the same species are analysed, only one of these may express the BGC in question. True transcriptional inactivity of BGCs may be due to a mutation in a structural or regulatory gene, although the former is less logical as the entire biosynthetic machinery would be produced in vain. An intriguing concept is that regulatory genes may sustain a single (frameshift) mutation, rendering the gene inactive, which may be easily restored by a compensatory mutation as a strategy for bet-hedging in a community, as seen for isolates of Streptomyces lunaelactis ${ }^{83}$. Such 'light-switch silencing' may be a primary mechanism by which strains are able to maintain large numbers of BGCs, as natural product biosynthesis is an energyintensive process. Still, it is reasonable to assume for many of the silent BGCs that the right conditions for their transcriptional activation are lacking when the strain is grown in the laboratory. the specialized metabolism of S. coelicolor A3(2), the model organism for antibiotic biosynthesis. For a long time, this organism was known to produce four antibiotics, namely actinorhodin (Act), calcium-dependent antibiotic (Cda), undecylprodigiosin (Red) and the plasmid-encoded methylenomycin (Mmy). When the genome of $S$. coelicolor was sequenced, many more BGCs were uncovered than originally anticipated, and the same was true for other model streptomycetes ${ }^{13,14,48}$. Renewed efforts for drug discovery led to the surprising discovery of yet a fifth antibiotic in S. coelicolor, called coelimycin P1 (REF. ${ }^{49}$ ), in a model organism that had been studied by thousands of scientists in hundreds of laboratories around the world. Moreover, a novel branch of the biosynthetic pathway of the model polyketide actinorhodin was shown to be activated by co-cultivation with the fungus Aspergillus niger ${ }^{50}$, and mass spectral imaging revealed substantial changes in the secreted metabolome during interaction of S. coelicolor with five other Actinobacteria $^{23}$. These examples illustrate the concept of cryptic biosynthetic pathways, namely those that have been identified in the genome but the cognate natural products of which are not synthesized under laboratory conditions (BOX 1). This concept has revolutionized the field of antibiotic research ${ }^{51}$, leading to the current era of genomics-based drug discovery $y^{3,4}$.

Nowadays, next-generation genome sequencing enables scientists to explore large numbers of microorganisms in search of novel BGCs. However, there are several challenges. A first challenge lies in prioritizing (cryptic) BGCs in terms of their potential for chemical novelty and/or clinical relevance to optimally exploit the wealth of available biological, genomic and metabolomics data. Second, the bulk of the microorganisms in soil and marine environments resist cultivation under laboratory conditions and thus represent a huge 'white space' of biochemical diversity ${ }^{52,53}$. Last, many BGCs are not expressed during laboratory cultivation. To unlock this potential, we need to better understand the ecological context in which Actinobacteria live, as this will provide clues to the mechanisms that activate the biosynthetic pathways of natural products. To leverage genome information to this end, it is of major importance to understand how such ecological forces shape actinobacterial genomes and how biosynthetic diversity evolves.

\section{Evolution of biosynthetic repertoires}

Vertical inheritance and horizontal gene transfer. The diversity of the environments that Actinobacteria inhabit is extraordinary ${ }^{2,54}$. Specialized metabolites have been isolated from marine organisms such as Salinispora spp..$^{55}$, arctic Streptomyces nitrosporeus ${ }^{56}$, desert-dwelling Streptomyces spp. ${ }^{57}$ and species that live in the microbiomes of animals, insects and plants ${ }^{30,57-59}$. This ecological diversity has major implications for the evolution of secondary metabolic repertoires, which are driven by a combination of vertical inheritance and horizontal gene transfer (HGT). In terms of vertical inheritance, Salinispora spp. show a strong relationship between phylogeny and BGC diversity, with close relatives sharing nearly all BGCs ${ }^{60}$. Similarly, in Amycolatopsis, BGCs are conserved within clades but not between them ${ }^{61}$. 

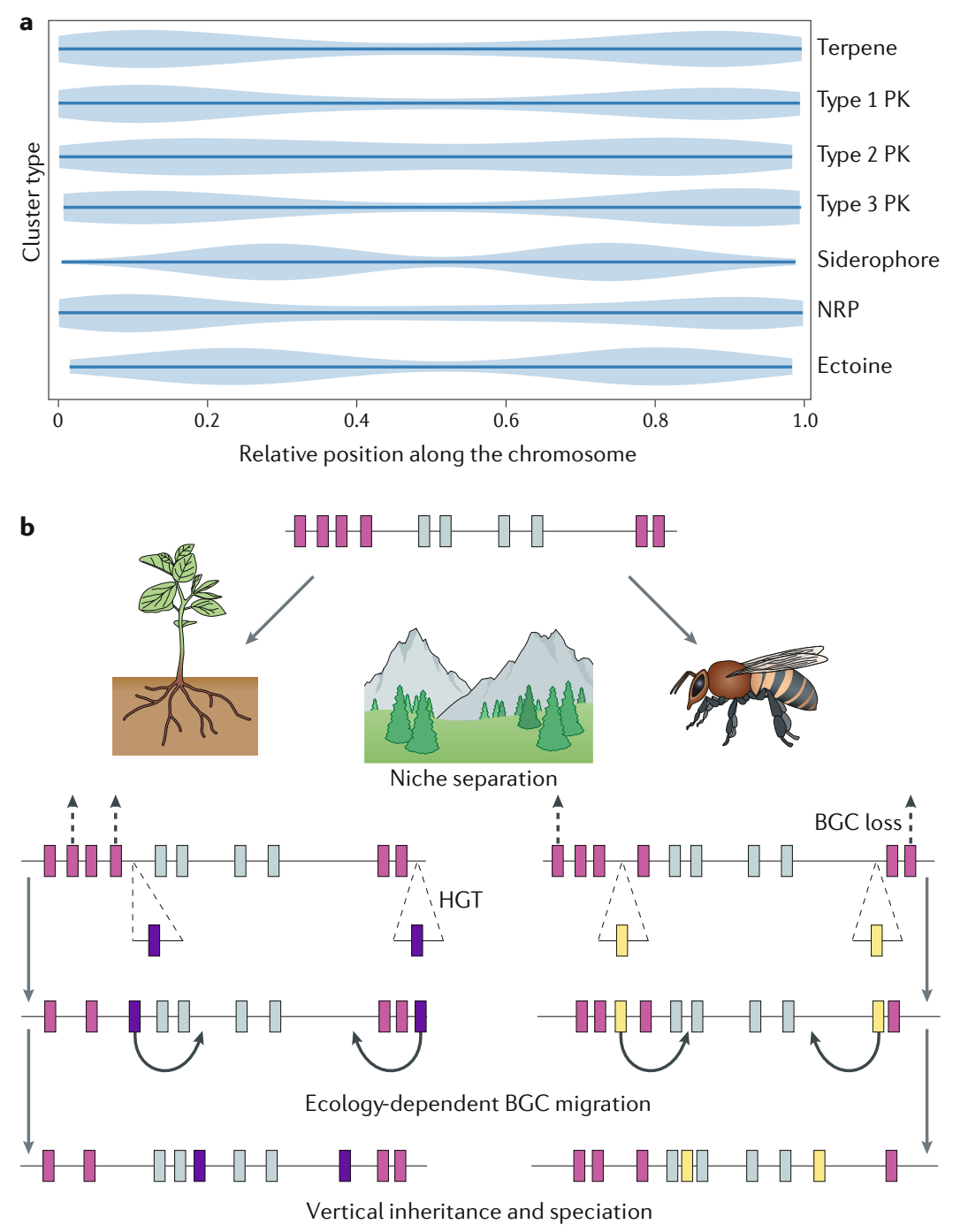

Fig. 2 | BGC distribution and evolution in Streptomyces chromosomes. a | Distribution of biosynthetic gene clusters (BGCs) encoding terpenes, non-ribosomal peptides (NRPs), polyketides (PKs), siderophores and ectoines in 100 linear Streptomyces chromosomes. Clusters encoding type $1 \mathrm{PKs}$, type $3 \mathrm{PKs}$ and NRPs localize to the chromosome ends, whereas clusters encoding type $2 \mathrm{PKs}$, terpenes, siderophores and ectoines localize more towards the chromosome core. $\mathbf{b}$ | Proposed movement of BGCs throughout evolution. Upon niche separation, genomes in different environments take up different genetic material through horizontal gene transfer (HGT) and/or BGCs are lost. In the long term, BGCs that are important for survival in that niche migrate towards the chromosome core and have a greater chance of being maintained through vertical inheritance.

Prodiginines

A family of red-pigmented,

bacterial, specialized

metabolites with a wide

range of biological activities,

including antibacterial,

antitumour and

immunosuppressive

properties.

Actinorhodin

A blue-pigmented polyketide

antibiotic that is a model for

polyketide biosynthesis.
These BGCs may be more important for adaptation than for survival. Similarly, rapid demographic expansion of Streptomyces into previously uninhabited niches has led to differentiation into various species clusters, distinguishable from each other by ancestral homologous recombination events ${ }^{62}$.

HGT is another important evolutionary driver of chemical complexity of natural products in Streptomyces ${ }^{60,63}$. In Salinispora, it has been estimated that up to $96 \%$ of the biosynthetic pathways may have been acquired through $\mathrm{HGT}^{60}$. It is unclear how frequent HGT occurs in Actinobacteria. Some argue that lateral acquisition and subsequent maintenance of complete BGCs is very rare $^{64}$. Nevertheless, it is likely that HGT has a key role in shaping BGC repertoires. Many more ancient HGT events of complete BGCs will have occurred than those that can still be reliably inferred, because those BGCs will have diverged over time since the moment of transfer ${ }^{65}$. The large discordance between gene phylogenies of core biosynthetic genes and species phylogeny testifies to this ${ }^{66-68}$. When HGT leads to acquisition of BGCs with similar functions, there may be strong evolutionary pressure for BGC loss. Indeed, in the evolutionary history of the genus Salinispora, genes for the biosynthesis of the siderophore desferrioxamine were lost in three strains independently as a direct consequence of HGT of a functionally similar BGC. Such events may happen at a large scale and therefore could mask a large proportion of historical HGT events ${ }^{69}$. In fact, rates of HGT may vary strongly between different types of BGCs, depending on their ecological roles; many species have a conserved 'core' set of BGCs, together with a strongly varying set of 'accessory' BGCs that is acquired or exchanged through $\mathrm{HGT}^{61,70}$. This is evident in Amycolatopsis, in which BGCs that were acquired through HGT largely localize to non-conserved genomic regions ${ }^{61}$.

Chromosome structure. Genomic structure is intimately tied with genetic change and conservation in specialized metabolism, possibly both as cause and effect. Actinobacteria have either linear or circular chromosomes. In linear chromosomes, as seen in Streptomyces and Rhodococcus, strain-specific genes and BGCs that are incorporated through HGT tend to be localized in the unstable subtelomeric end regions of the chromosome ${ }^{63,71-73}$. Selective pressure might induce migration of such BGCs towards the chromosomal core, where they are likely more stably maintained through vertical inheritance and thus display much higher levels of conservation across different actinobacterial species ${ }^{71-73}$. A recent heterologous expression study also showed that the chromosomal location of BGCs has an effect on expression levels, with expression levels of a $\beta$-glucuronidase reporter gene measured highest in the central regions of the chromosomal $\mathrm{arms}^{74}$. In Streptomyces, more centralized BGCs tend to encode molecules such as ectoines and siderophores (FIG. 2), which are likely to be essential for survival of the genus. In the circular chromosomes of Salinispora and Amycolatopsis, species-specific BGCs are largely located on genomic islands relatively distant from the origin of replication ${ }^{75}$. Conversely, conserved BGCs tend to be localized in the 'core genome'. As in Streptomyces, in Amycolatopsis these BGCs specify metabolites, such as ectoines, siderophores and terpenes, whereas strain-specific BGCs, which make up as much as $67 \%$ of all BGCs in Amycolatopsis, localize largely away from the core genome to the genomic island ${ }^{61}$. Although, to our knowledge, no studies have investigated BGC migration from peripheral regions to core regions, $\mathrm{BGC}$ migration between genomic islands has been inferred to be likely based on phylogenomic analyses, which supports the hypothesis that BGCs preferentially migrate towards genomic core regions $\mathrm{s}^{75}$.

One of the reasons why actinobacterial genomes are packed with BGCs is because there are well-established mechanisms in place to acquire and exchange BGCs. 


\section{Ectoines \\ Bacterial specialized \\ metabolites that act as \\ osmolytes and protect against \\ osmotic stress induced by, for \\ example, changes in salt \\ concentration of the \\ environment. \\ Terpenes \\ A class of specialized \\ metabolites produced by \\ plants, fungi and bacteria with \\ different biological activities, \\ often volatile and with a strong \\ odour.}

One common method of acquisition is through integrative and conjugative elements (ICEs). These ICEs are plasmids with the ability to integrate themselves into the chromosome. The prevalence of these ICEs seems to be partially dictated by their ecological background: Actinobacteria originating from soil, plants or aquatic environments contain a greater number of ICEs than species from other environments ${ }^{76}$. In addition to ICEs, giant linear plasmids have a high density of BGCs for antibiotics, which suggests an important role in the acquisition of bioactive metabolites throughout evolution ${ }^{77-79}$.

\section{Control of antibiotic production}

Just as ecological forces shape the organization of BGCs across actinobacterial chromosomes, they also shape how the clusters are regulated. A range of external signals influence production either directly, through activation of pathway-specific activators, or indirectly, via an interactive network of pleiotropic regulators and intracellular signalling molecules ${ }^{44,80,81}$. Additionally, cross-regulation can exist between different BGCs, as was recently shown for the two chemically unrelated specialized metabolites antimycin and candicidin produced by Streptomyces albus $\mathrm{S} 4$. Their BGCs are separated by $9 \mathrm{~kb}$ on the chromosome, but the pathway-specific regulator of candicidin, FscR1, can bind directly upstream of the genes encoding antimycin biosynthesis and is essential for the activation of this cluster ${ }^{82}$. This regulatory complexity is highlighted by the strong emphasis on regulation in the genome of the model organism $S$. coelicolor, which encodes close to 800 regulatory proteins, representing $>10 \%$ of the total proteome ${ }^{13}$. Higher-level control is likely to be tied to the ecological conditions in which these adaptive responses have evolved. Environmental and physiological signals have been integrated into the regulation of specialized metabolism to ensure that these costly molecules are only produced when required ${ }^{2,80}$. The involvement of environmental signals in the control and complexity of specialized metabolism is illustrated by the control of the BGC for ferroverdins and bagremycins in Streptomyces lunaelactis ${ }^{83}$. The metabolite produced from the BGC depends on iron availability. In iron-limiting conditions, the antimicrobial bagremycins are produced. When there is an excess of iron, the amino group of bagremycin is replaced by a nitroso group to generate ferroverdin, a siderophore that is used as an anticholesterol drug and that, in nature, likely functions to limit iron-mediated oxidative damage ${ }^{83}$.

In a laboratory setting, the absence of the triggers and cues that would activate antibiotic production in the original habitat offers a possible explanation for why so many BGCs remain poorly expressed or silent under laboratory growth conditions (BOX 1). However, the identity of environmental ligands and/or signals perceived by both pleiotropic and pathway-specific regulatory proteins is a major area of investigation and if resolved could lead to the activation of silent BGCs and thus drug discovery ${ }^{44}$. The key lies in understanding the biology of the producing bacteria and translating these insights into solutions to activate antibiotic production (see below).
Here, we provide some background on the complex transcriptional control of BGCs and then look into the regulatory networks that control the well-established connection between the onset of development and antibiotic production. For detailed reviews, we refer the reader elsewhere ${ }^{43,44,84}$.

Principles of the control of antibiotic production. To coordinate the metabolic responses to specific ecological challenges, Actinobacteria have evolved complex multilevel regulatory networks. These networks are composed of multilevel transcriptional and translational control. This control is required for the correct interpretation of the signals that reach the colony and to translate them into appropriate responses. Much of our knowledge of the control of antibiotic production has been obtained from the study of the BGCs for Act, Cda and Red in S. coelicolor and for streptomycin in Streptomyces griseus ${ }^{43,44,84}$. These clusters are controlled by the pathway-specific activators ActII-ORF4, CdaR and RedD, which belong to the SARP family of Streptomyces antibiotic regulatory proteins $^{85}$, and StrR (ParB-Spo0J family ${ }^{86}$ ), respectively. These cluster-situated regulators directly control the level of transcription of the BGC, which in turn dictates the production level of the cognate natural product ${ }^{87,88}$. Interestingly, actII-ORF4, $c d a R$ and redD are all subject to translational control by the tRNA that recognizes the rare UUA codon for leucine. This tRNA, encoded by the bldA gene, is also required for the proper translation of many developmental genes and thus links morphological to chemical differentiation ${ }^{89,90}$.

Multiple cluster-situated regulators may control a single BGC, and in addition the BGCs are subject to global control. This enables the cell to coordinate specialized metabolism with growth and development, the balance in carbon, nitrogen and phosphorus metabolism, and other major cellular pathways, thereby generating a complex hierarchy of regulatory networks. To enable efficient responses to external stimuli, the activity of many regulatory proteins is determined by small molecules. This includes the hormone-like $\gamma$-butyrolactones ${ }^{91,92}$, feedback through biosynthetic intermediates ${ }^{93-95}$ and sugar-based ligands ${ }^{96,97}$. The identification of such external signals, often referred to as elicitors, is of key importance for the rational activation (elicitation) of natural product biosynthesis and thus for the revitalization of drug discovery.

PCD and the DasR regulatory network. As described above, PCD mediates the provision of nutrients at the onset of morphological and chemical differentiation. The signalling pathway from PCD to differentiation revolves around the global nutrient sensory GntRfamily regulator DasR (FIG. 1). Autolytic degradation of cell-wall peptidoglycan releases the amino sugars $\mathrm{N}$-acetylglucosamine (GlcNAc) and $\mathrm{N}$-acetylmuramic acid (MurNAc) around the colonies. Under nutrientlimiting conditions (famine), the accumulation of GlcNAc around colonies triggers development and antibiotic production, whereas under rich growth (feast) conditions, GlcNAc blocks both processes ${ }^{97}$. The rationale behind this is that under feast conditions 


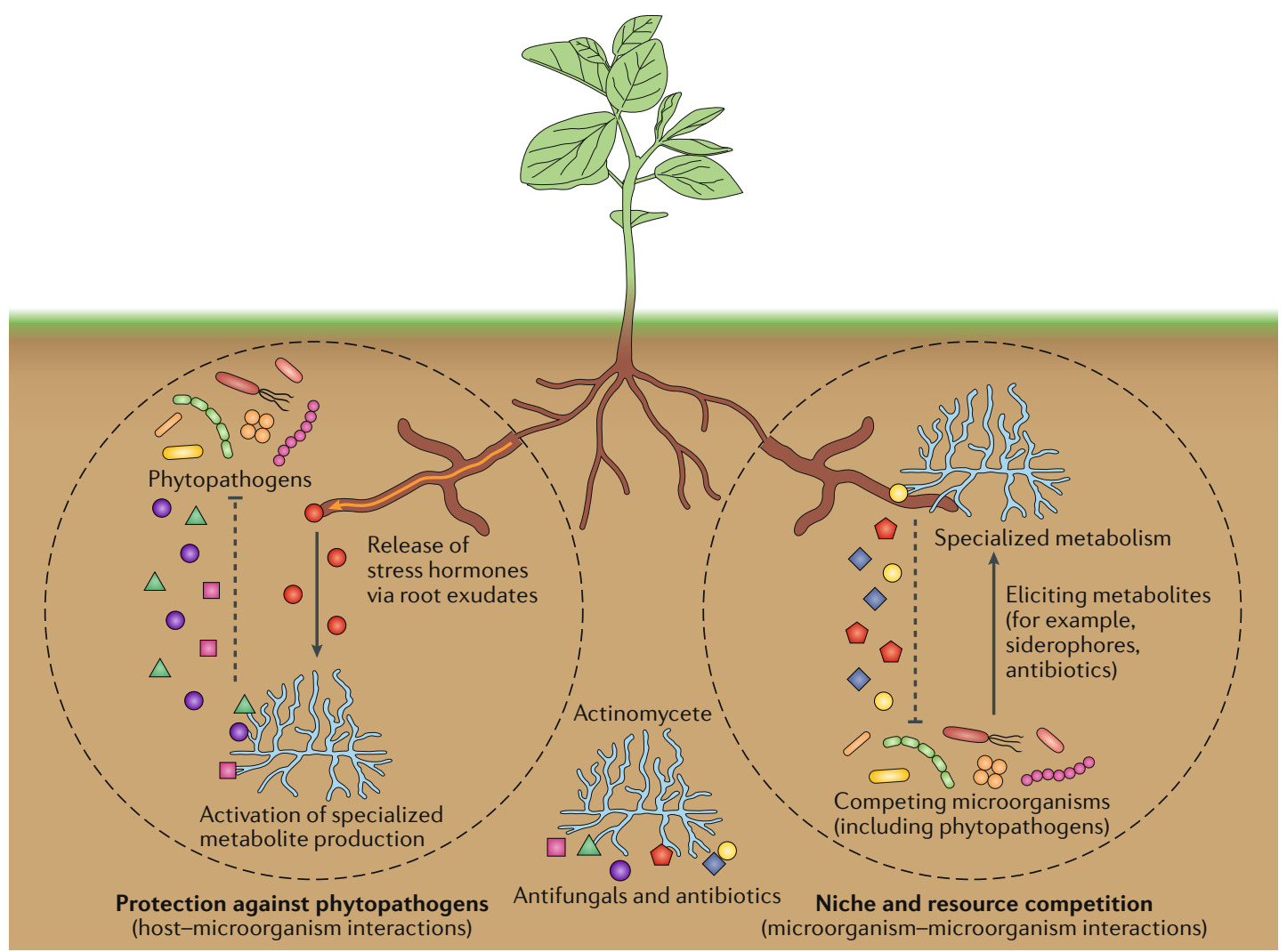

Fig. 3 | Natural products of Actinobacteria in host-microorganism and microorganism-microorganism interactions. Within the natural habitat of Actinobacteria, environmental signals are likely to have a key role in the activation of specialized metabolism. Chemical ecological interactions can, for example, be found in the rhizosphere of plants. Upon stress, plants release stress hormones (for example, jasmonic acid) via their root exudates. These hormones can activate antibiotic production by Actinobacteria, which can protect the plant against phytopathogens ('cry for help'). Additionally, competitive interactions occur between different members of the microbial soil community (both symbionts and pathogens). Metabolites, such as siderophores and antibiotics, from the competing microbial community can elicit changes in the metabolic profile of Actinobacteria, including antibiotic production. These molecules have an important role in shaping the rhizosphere microbiome and inhibiting (opportunistic) pathogens, thus also protecting the host.

GlcNAc is seen as derived from chitin and signals nutrient abundance and promotes growth, whereas under famine conditions it signals hydrolysis of the bacterial cell wall and thus the need for development. GlcNAc-derived glucosamine-6-phosphate (GlcN-6P) and other phospho-sugars act as ligands for DasR, and thereby inactivate the repressor, which results in the derepression of $\mathrm{BGCs}^{97}$. DasR directly controls the pathway-specific activators of BGCs for all antibiotics and siderophores in S. coelicolor ${ }^{98-100}$. Addition of GlcNAc under nutrient-limiting conditions activates the transcription of antibiotic BGCs, including $c p k$ which specifies the cryptic polyketide coelimycin; this principle is now also being applied in industrial screening regimes. Interestingly, there is direct competition between the DasR regulon and the regulatory networks governed by the transcription factors AtrA and Rok7B7: DasR represses actII-ORF4 and nagE2, encoding the activator of Act biosynthesis and the GlcNAc transporter, respectively, whereas these genes are activated by (and depend on) Rok7B7 and AtrA ${ }^{101}$. This system highlights the complex control of bioactive molecules in response to environmental changes. It also illustrates the value of discovering the ecological rationale behind antibiotic production and using such knowledge to activate antibiotic production (FIC. 1).

Identifying the elicitors that activate cryptic BGCs. Various other approaches have been developed to identify the environmental signals involved in the regulation of actinobacterial specialized metabolism. The signals that trigger the expression of BGCs act through a transcriptional regulatory network, governed via cis-regulatory elements targeted by transcription factors. Many transcription factors will respond to ligands, but how do we uncover what these ligands are? Genomic context is one major pointer. For example, if a regulatory gene lies next to a metabolic operon (such as for sugar metabolism), this may be an important clue. Additionally, transcription factors are often autoregulatory, and the cis-regulatory element is therefore typically found in the upstream region of the gene. With the cis-regulatory element in hand, computational approaches can then be used to predict the regulatory network in silico. In the case of DasR, it was immediately obvious that the best hits in those predictions all related to GlcNAc metabolism or transport, and identifying glucosamine-6P as the ligand was then fairly straightforward ${ }^{97}$. Methods directed at single 


\section{Box 2 | Host-derived signals as elicitors}

The high abundance of Actinobacteria in plant microbiomes, specifically members of the genus Streptomyces, suggests that these bacteria are actively recruited by plants, possibly via root exudates ${ }^{158-160}$. Plants exudate up to $30-60 \%$ of their photosynthate into the rhizosphere, which contains large quantities of sugars and amino acids that can influence the growth of beneficial bacteria as well as their antibiotic production (for example, through catabolite repression) $)^{2,130,158}$. These exudates contain plant metabolites, such as the phytohormone salicylic acid, which has been positively correlated with the abundance of operational taxonomic units attributed to Streptomycetaceae in the soil, as well as endophytic Actinobacteria ${ }^{159}$. As such hormones are released under pathogenic stress, an interesting hypothesis is that exudation of such compounds may be a means to 'cry for help': the release of stress hormones by the host results in the recruitment of antimicrobial-producing Actinobacteria to reduce the severity of pathogenic infection ${ }^{2,161}$ (FIG. 3). Interestingly, when Actinobacteria are grown in the presence of plant stress hormones, such as jasmonic acid and salicylic acid, changes in the production of specialized metabolites are observed, often leading to increased antimicrobial activity ${ }^{13}$ (A. van der Meij, J.M. Raaijmakers and G.P.v.W., unpublished observations). This could indicate that evolved regulatory networks exist between the host and members of its microbiome, opening up the intriguing possibility to design microorganisms that produce bioactive molecules specifically in response to stress induced by pathogen-derived signals. We hypothesize that similar use of Actinobacteria as 'medicine producers' may happen in our own microbiome, with mammalian stress hormones as activators of specialized metabolism of Actinobacteria during infection.

bacterial producer strains include varying the composition of growth media ${ }^{102,103}$, inducing antibiotic resistance ${ }^{104,105}$ and microbial co-cultivation ${ }^{106-108}$. Screening for new chemical elicitors of antibiotic production is a promising approach, as this can enhance the chance of success in high-throughput screening of bacterial strain collections. Logical elicitors to include in such screens are those with a proven pleiotropic activity, such as $\mathrm{GlNAc}^{97}$, $\gamma$-butyrolactones ${ }^{92,109}$ and histone deacetylase inhibitors ${ }^{110}$. Screening compound libraries for small molecules that perturb antibiotic production was shown to be an effective strategy to identify novel elicitors of antibiotic produciton ${ }^{111}$. Another example is bioactivity highthroughput elicitor screening technology, in which a wild-type microorganism is subjected to a library of small molecules, and the resulting induced metabolomes are screened for bioactivity against a chosen indicator strain $^{112,113}$. Use of this method led to the identification of various cryptic antibiotics, including the novel lanthipeptide cebulantin ${ }^{112}$ and the novel naphthoquinone epoxide hiroshidine ${ }^{113}$. The method also identified atenolol, a $\beta$-blocker clinically used to treat hypertension, as a global elicitor ${ }^{113}$.

Chemical ecological relationships as elicitors of antibiotic production. Within their natural environment, Actinobacteria are part of diverse microbial communities that include archaea, bacteria, fungi, protists and viruses. Within these communities, specific interactions have evolved, and small molecules, such as specialized metabolites, facilitate interactions between different microbial species (symbionts or competitors), including the activation of antibiotic production ${ }^{14,115}$. By mimicking these naturally occurring chemical-ecological relationships in so-called co-culture experiments, cryptic BGCs might be activated in the laboratory.

Indeed, co-cultivation of Actinobacteria with other bacteria or fungi changes their specialized metabolite production profile. Examples include the production of alchivemycin A by a Streptomyces strain following co-cultivation with the mycolic acid-producing Tsukamurella pulmonis ${ }^{116}$, biosynthesis of a range of metabolites during co-culture of Aspergillus nidulans and various streptomycetes ${ }^{117}$, and the activation of a silent pathway of actinorhodin in S. coelicolor upon co-cultivation with $A$. niger ${ }^{50}$. Co-culture of marine-derived Streptomyces spp. with different human pathogens, including methicillin-resistant Staphylococcus aureus, resulted in increased production of different antibiotics and enhanced biological activity ${ }^{118}$. Co-culture with multidrug-resistant bacteria might emerge as an effective, targeted approach to find novel bioactive compounds with activity against the pathogens for which new antibiotics are desperately needed.

The signals and cues that mediate the observed changes in specialized metabolite production are diverse and include physical cell-cell interactions ${ }^{116,119}$, a higher rate of nutrient depletion ${ }^{120}$, enzymatic conversion of precursors to active metabolites ${ }^{121}$, HGT $^{122}$ and microbial small molecules ${ }^{23,123,124}$. However, for many interactions, the signals - and specifically the molecular mechanisms - are as yet unknown. The development of analytical techniques such as nanospray desorption electrospray ionization and MALDI-TOF imaging mass spectrometry enables the direct visualization of molecules exchanged during the chemical communication between microorganisms ${ }^{125}$. These methods might help with the elucidation of the signals involved in above-described interactions.

Actinobacteria are also found in close association with various eukaryotic hosts (FIG. 3), and there are multiple examples of defensive symbioses between Actinobacteria and the host, in particular for plants (suppressive soils) and insects (fungus-growing ants) ${ }^{54,126,127}$. An interesting example is provided by leafcutter ants, which live in symbiosis with the fungus Leucoagaricus and with Pseudonocardia bacteria ${ }^{127}$. The Pseudonocardia produce bioactive compounds to protect the fungal cultivar against infection by other fungi. Recent work showed that, in return, the presence of Pseudonocardia elicits the production of antimicrobials by pathogenic Escovopsis fungi during infection of the cultivar ${ }^{128}$. This exemplifies an evolutionary arms race between the Actinobacterium and the fungus.

Interestingly, many plant growth-promoting bacteria produce phytohormones, such as auxin and gibberelic acid ${ }^{129}$. This suggests that the host and the microorganism communicate (or hijack each other's communication channels) through the production of such metabolites. This may indeed work both ways, as plant hormones influence growth and specialized metabolism by endophytic Actinobacteria ${ }^{130}$ (A. van der Meij, J.M. Raaijmakers and G.P.v.W., unpublished observations), which reveals that host-specific signals can also affect specialized metabolism (BOX 2).

\section{Genome mining strategies}

The biosynthetic diversity found in Actinobacteria is enormous. Genomic data sets have become increasingly larger, with massive strain collections, pan-genomes and metagenomes sometimes containing 
genetic information for thousands of Actinobacteria at once. A range of computational tools (for example, antiSMASH ${ }^{131}$ or PRISM $^{132}$ ) have been developed in the past decade that automate the identification of BGCs in these genomes and, to a certain extent, facilitate prediction of the structures of their products (BOX 3).

Given the increasing size of genomic or metagenomic data sets, studying BGCs on a case-by-case basis is often no longer feasible. To address this problem, sequence similarity networking approaches have been developed to automatically relate predicted BGCs to gene clusters of known function (from, for example, the MIBiG database $^{133}$ ) and to group them into gene cluster families (GCFs) ${ }^{32,60,134]}$. The members of a GCF are then predicted to produce the same or highly similar molecules. Using their streamlined computational framework for BGC similarity networking, researchers recently studied 3,080 actinobacterial genomes and found that they contained around $\sim 18,000$ distinct GCFs, the vast majority of which have no known products ${ }^{68}$. This constitutes an enormous potential for discovery and to some extent enables us to differentiate between BGCs that are likely to produce compounds we have seen before and BGCs that may encode novel chemistry. However, the number of GCFs to which no known functions or chemistries can be linked is so great that it is difficult to know which of the BGCs belonging to them encode the production of the pharmaceutically most interesting molecules.

Some advances have been made to predict the functions of BGCs. For example, the Antibiotic Resistance Target Seeker $\left(\right.$ ARTS $^{135}$ ) prioritizes BGCs based on co-localization with resistance genes. The rationale behind this is that bacteria need a mechanism to protect themselves against the antibiotic molecules they are producing. Therefore, resistance markers may function as beacons to prioritize specific BGCs for antibiotic discovery. Unfortunately, only a small percentage of BGCs

\section{Box 3 | Computational tools for genome mining of biosynthetic diversity}

The most commonly used tool for predicting biosynthetic gene clusters (BGCs) in bacterial genomes is antiSMASH. Based on core genes, antiSMASH predicts the gene cluster type (non-ribosomal peptide, polyketide, terpene, siderophore and so forth). It also annotates and groups accessory genes and minimally predicts the specialized metabolite core scaffold, enabling researchers to quickly identify BGCs of interest in an uploaded genome ${ }^{131}$. For instance, antiSMASH output was used as the starting point for a phylogenetic prioritization method leading to the discovery of the novel compound corbomycin $^{162}$. Other BGC identification tools also exist, such as BAGEL and PRISM ${ }^{132,163}$. The database MIBiG, which charts known BGCs and their products, provides a means of easily comparing predicted clusters with experimentally characterized ones that have known chemical products ${ }^{133}$.

For BGC mining on a larger scale, the networking tool BiG-SCAPE can be used to cluster both full and fragmented BGCs into gene cluster families, to obtain a comprehensive overview of BGC diversity in large genome collections or metagenomes. In such large data sets, the tool CORASON can help with prioritizing BGCs of interest by providing insights into the evolutionary context of BGCs through phylogenetic analysis ${ }^{68}$. Genome mining approaches can also be combined with proteomics for more efficient prioritization ${ }^{164}$. Additionally, metabolomics data can be coupled to structure predictions yielded by genomics data or to absence-presence patterns of BGCs, in order to link molecules to $B G C s^{165,166}$. The power of these methods will only increase now that tools are able to predict the function of natural products from their (predicted) structures ${ }^{167}$. As structure prediction is easier on a substructure level, there are ongoing efforts to attempt linking substructure predictions to mass shifts, which can be a great aid in elicitation studies and dereplication ${ }^{147}$. have distinguishable self-resistance markers. Ecological insights are needed to provide complementary strategies to know which microorganisms are most likely to encode biosynthetic pathways of interest, which BGCs among these are functionally most desirable and how they can likely be activated.

Where to find chemical novelty. To effectively mine Actinobacteria for drug discovery, there is a need for guidance towards chemical novelty. First of all, this requires a better understanding of the environmental and taxonomic distributions of BGCs. Such knowledge can help estimate where to search for novel producers and whether this search should be based on phylogeny, geography or specific environmental niches ${ }^{31,61,134}$. Taxonomic groups that are particularly gifted in terms of their natural product diversity include Streptomycetales, Streptosporangiales, Frankiales, Micromonosporales and Pseudonocardiales ${ }^{134}$. To decrease the risk of rediscovery of known molecules, known as replication, focus is directed towards rare Actinobacteria, of which many taxa have been greatly underexplored. Indeed, genera such as Micromonospora, Amycolatopsis, Salinispora, Nocardia and Verrucosispora are a source of chemically unique metabolites with potent antibacterial activities, such as abyssomycins and proximicins ${ }^{136}$. Besides phylogeny, geographic location has also been proposed as an indicator of BGC distribution ${ }^{137}$. However, comparative genomics of Amycolatopsis and Salinispora strains both show that taxonomy is a more important indicator of BGC distribution than geographic location ${ }^{55,60,61}$. Intriguingly, regardless of their geographic origin, strains of Salinispora arenicola all produced rifamycin, staurosporine and saliniketal ${ }^{55}$, suggesting that, independent of the niche, strains may produce the same molecules or analogues. To a certain degree, "everything is everywhere and the environment selects", as Dutch microbiologist Baas-Becking proposed almost a century ago. Indeed, a study of biosynthetic diversity in soils from Central Park in New York City, United States, suggests that the degree of novelty found in common areas may be similar to that in more exotic locations ${ }^{138}$.

Still, exploration of extreme or unusual environments, such as hyper-arid deserts, permafrost soils, mangrove trees, caves and deep-sea sediments that are characterized by challenging conditions (aridity, high salinity, low nutrient sources and extreme temperatures), showed high diversity of BGCs ${ }^{57,139}$. Between 2010 and 2018 alone, taxonomically diverse microorganisms originating from extreme environments have been the source of nearly 200 new specialized metabolites, many of which were produced by Actinobacteria ${ }^{57}$. Microorganisms from the permafrost soil synthesize a broad range of chemical compounds ${ }^{136}$. Other interesting sources of gifted Actinobacteria are the microbiomes of diverse eukaryotic hosts, including insects, sponges and humans $s^{58,59,140}$. Within microbiomes, pathogen pressure selects for Actinobacteria that produce efficacious and relevant antimicrobials. Furthermore, this host association could potentially enrich for compounds with low toxicity to animals. This makes host microbiomes a promising source of novel molecules, 


\section{REVIEWS}

a
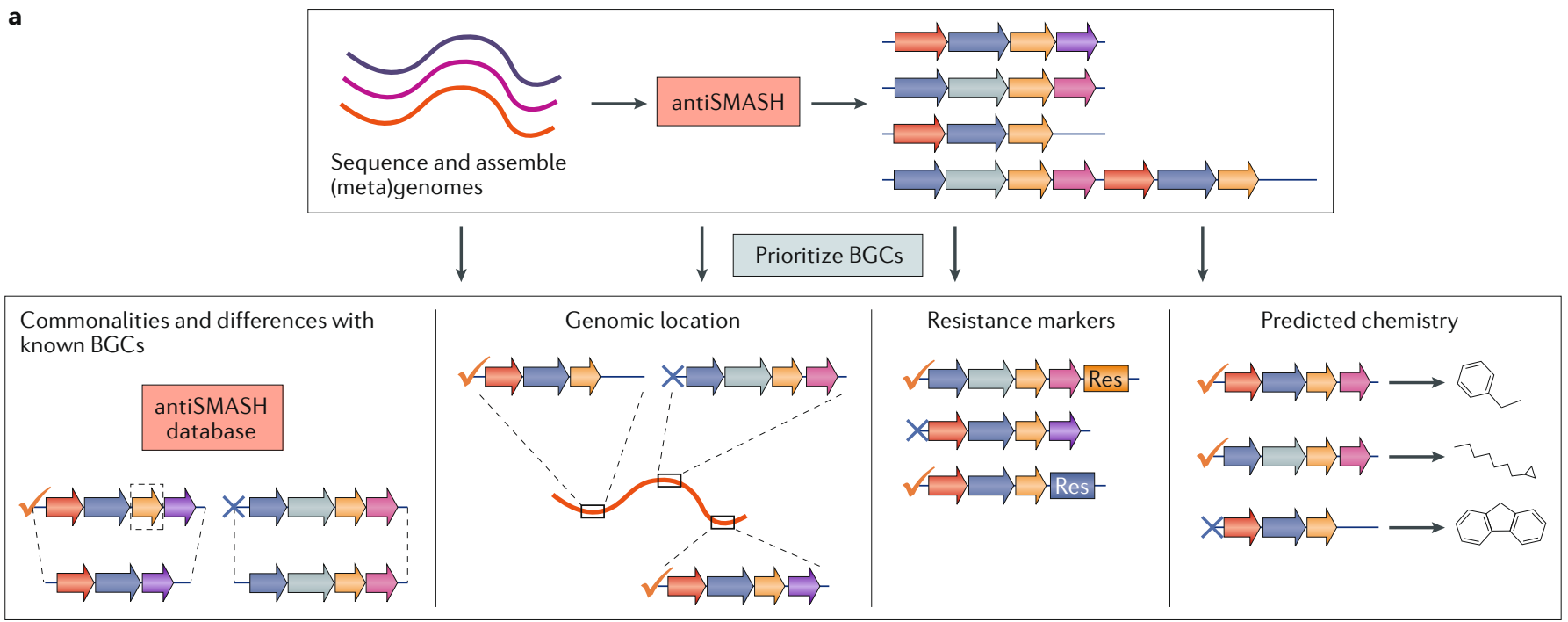

Candidate BGCs

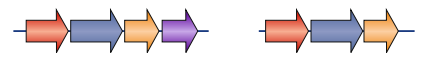

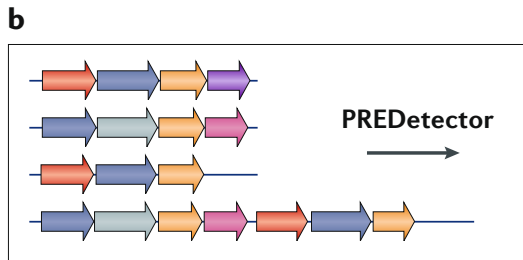

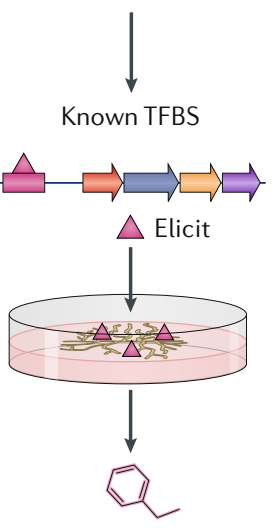

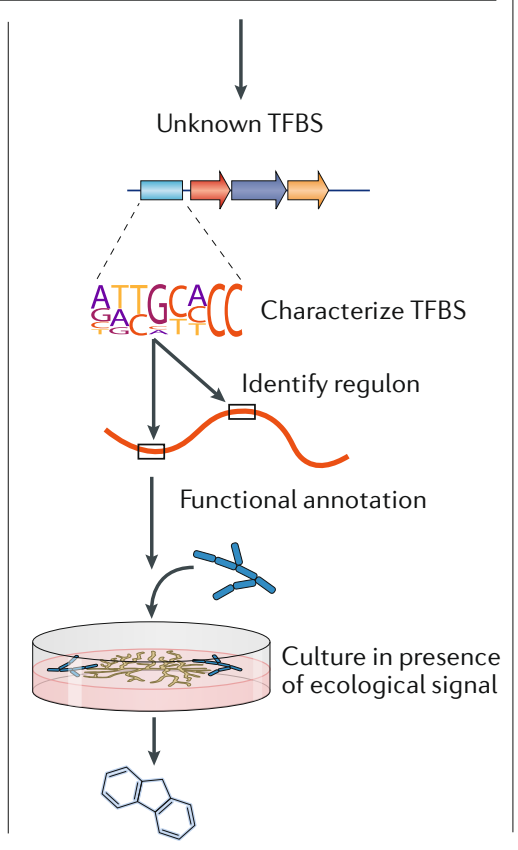
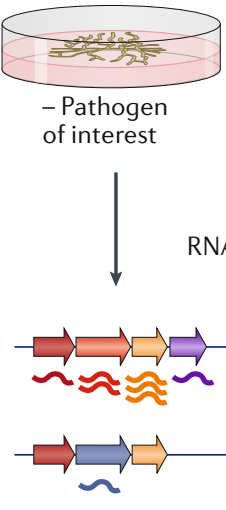

c

Co-cultures

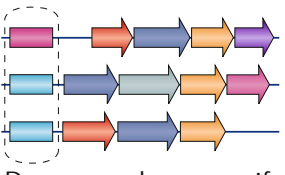

etect regulatory motifs

with possibly a higher potential to be successfully used in the clinic. Metabolomic analysis of Streptomyces spp. from insect microbiomes displayed immense potential for novel chemistry in these strains ${ }^{58}$. The same study demonstrated how principal component analysis can be leveraged for strain prioritization; a strain characterized as a metabolic outlier produced cyphomycin, a novel antifungal agent active against multidrug-resistant fungi ${ }^{58}$.

However, we should remind ourselves that even well-studied organisms such as S. coelicolor still harbour undiscovered biosynthetic pathways. For instance, it was shown that inactivation of the biosynthetic genes for the common antibiotics streptothricin and streptomycin 
4 Fig. 4 | Omics strategies for BGC prioritization and elicitation. a | Genomics-based prioritization of biosynthetic gene clusters (BGCs). After identifying putative BGCs through antiSMASH, BGCs can be prioritized based on commonalities and differences with known BGCs, their location on the genome, resistance markers in the vicinity of the $\mathrm{BGC}$ and predicted chemistry of the compound that the BGC produces. $\mathbf{b} \mid$ Genomicsbased prediction of BGC regulatory sequences and elicitors. After predicting both known and unknown transcription factor binding sites (TFBS) with PREDetector, species with known TFBS can be directly elicited. Unknown TFBS can be characterized by generating a sequence profile of similar TFBS and searching the genome for the entire regulon regulated by the corresponding transcription factor. From this, the genes in the regulon can be functionally annotated, and the ecological signal that triggers BGC expression can be inferred. $\mathbf{c}$ | Transcriptomics-based prioritization of BGCs. Differential gene expression of BGCs can be quantified through RNA-sequencing (RNA-Seq) of co-cultures or microbiomes with or without a pathogen of interest. BGCs that are expressed when the pathogen of interest is present are more likely to have a role in targeting this pathogen and host protection. identity of the global regulator can potentially be very informative about the ecological function of the BGC and therefore the function of its product. For example, in a plant microbiome setting, BGCs regulated by DasR are likely to respond to GlcNAc, which is also a breakdown product of fungal cell walls; hence, this could point to a possible antifungal role of a compound produced by such a BGC. Computational searches have the potency to identify the entire regulons associated with them ${ }^{142,143}$, and the gene content of these regulons may provide valuable data on the ecological functions of this regulon and by proxy of the BGC in question ${ }^{144}$. The specific molecules eliciting the activation of these regulons would then still need to be identified; potentially, paired metabolomics and metatranscriptomics of native communities where the Actinobacterium resides may provide means to identify which molecules are specifically present when expression of the BGC is triggered. Such predicted regulatory cues can in turn feedback into tools such as PREDetector to find novel BGCs in other species that may be similarly elicited.

Furthermore, using metatranscriptomic data (or transcriptomic data of co-cultures) is likely to be a powerful technology to predict the roles of BGCs in interaction with other organisms (FIG. 4C). Knowing under which conditions members of certain GCFs are expressed can illuminate their likely functions and hint towards how they may be regulated. For example, determining which bacterial BGCs in a plant endosphere microbiome were upregulated upon fungal infection recently led to the identification of a gene cluster essential for disease suppression ${ }^{145}$. The attractive concept that chitooligosaccharides produced from hydrolysis of the fungal cell wall elicit the production of the antifungal needs to be tested. Additionally, expression of BGCs can be correlated to the absence/presence/abundance of specific other organisms in the community, to identify whether they might either be triggered by their presence or effectuate their loss from the community by, for example, antibiosis. Adding metabolomics to the equation may provide further means of prioritization, as candidate products for a BGC can be identified that specifically appear when it is expressed, and tandem mass spectrometry analysis algorithms ${ }^{146-150}$ can be used to dereplicate them and predict (parts of) their structures to assess their novelty (BOX 3).

\section{Synthetic biology approaches to express gene clusters.}

Although computational strategies can be used to prioritize the most novel BGCs for experimental characterization, we then face the challenge of identifying the cognate chemical products of these BGCs. This currently remains a bottleneck, as thousands of potentially interesting BGCs can be found across publicly available genome sequences. By direct sequencing of environmental DNA, metagenomics even enables us to predict the chemical space of microbial 'dark matter': thus far uncultivated bacteria, which represent a promising source of novel natural products ${ }^{151}$. Synthetic biology is a powerful strategy to facilitate expression of BGCs observed in genome or metagenome sequences ${ }^{152}$. This strategy is illustrated by recent work on the 
identification of bioactive molecules from the human microbiome ${ }^{153}$. The synthesis of a metagenomic BGC and subsequent heterologous expression in S. albus enabled the isolation and identification of new polyketides, designated metamycin $\mathrm{A}$, metamycin $\mathrm{B}$, metamycin $\mathrm{C}$ and metamycin D. Advances in synthetic biology and genome engineering (reviewed in detail in REF. ${ }^{152}$ ) can become very useful in the expression of cryptic BGCs and the identification of their chemical products. However, designing a DNA sequence for a large pathway that will be functional in a model production host has remained more challenging than previously anticipated, as achieving the required stoichiometry between transcriptional units in a BGC for it to produce fully elaborated products is non-trivial ${ }^{154}$ : the required precursors or cofactors may be lacking in the heterologous host ${ }^{155}$, and low production titres from synthetic BGCs may hamper chemical characterization. Although all of these bottlenecks are being addressed, understanding the regulatory mechanisms behind BGC expression for now remains of key importance for the identification of their products. Once high-throughput refactoring of complex BGCs becomes a reality, we anticipate that ecological and regulatory information will be crucial to predict BGC functions and thus prioritize them for synthesis and expression.

\section{Conclusions and future perspectives}

Despite our advances in niche exploration revealing great potential for drug discovery, the current state of knowledge regarding BGC diversity and distribution in terms of ecology and phylogeny limits our ability to guide drug discovery. It is therefore necessary to further characterize the extant microbial diversity from different ecological niches and create a global survey of niche-correlated natural product diversity. Moreover, characterizing their functions in their native microbial communities, as well as their modes of action, will be crucial to advance our understanding of the regulation of specialized metabolism and hence for our effective ability to prioritize BGCs and elicit their expression. New technologies will be required for this, and in the 'omics' area we specifically envision a larger role for transcriptomic and metatranscriptomic studies of specialized metabolism, as well as regulatory network reconstruction, targeted to the most relevant microbiomes and ecological niches. Once we better understand which cellular and ecological conditions induce the expression of BGCs, this will greatly facilitate prioritizing gene clusters that are likely to have functions of interest and to predict which molecular stimuli are likely to activate them.

Published online 1 June 2020
1. Whitman, W. B. et al. Bergey's Manual of Systematic Bacteriology: Volume 5: The Actinobacteria (Springer Science \& Business Media, 2012).

2. van der Meij, A., Worsley, S. F., Hutchings, M. I. \& van Wezel, G. P. Chemical ecology of antibiotic production by actinomycetes. FEMS Microbiol. Rev. 41, 392-416 (2017)

3. Barka, E. A. et al. Taxonomy, physiology, and natural products of Actinobacteria. Microbiol. Mol. Biol. Rev. 80, 1-43 (2016)

4. Bērdy, J. Bioactive microbial metabolites. J. Antibiot. 58, 1-26 (2005)

5. Hopwood, D. A. Streptomyces in Nature and Medicine: the Antibiotic Makers (Oxford Univ. Press, 2007).

6. Vrancken, K. \& Anne, J. Secretory production of recombinant proteins by Streptomyces. Future Microbiol. 4, 181-188 (2009).

7. Newman, D. J. \& Cragg, G. M. Natural products as sources of new drugs over the last 25 years. J. Nat. Prod. 70, 461-477 (2007).

8. Davies, J. \& Davies, D. Origins and evolution of antibiotic resistance. Microbiol. Mol. Biol. Rev. 74 , 417-433 (2010).

9. Cooper, M. A. \& Shlaes, D. Fix the antibiotics pipeline Nature 472, 32 (2011).

10. Payne, D. J., Gwynn, M. N., Holmes, D. J. \& Pompliano, D. L. Drugs for bad bugs: confronting the challenges of antibacterial discovery. Nat. Rev. Drug. Discov. 6, 29-40 (2007)

11. Baltz, R. H. Antimicrobials from actinomycetes: back to the future. Microbe 2, 125-131 (2007).

12. Baltz, R. H. Renaissance in antibacterial discovery from actinomycetes. Curr. Opin. Pharmacol. 8, 557-563 (2008)

13. Bentley, S. D. et al. Complete genome sequence of the model actinomycete Streptomyces coelicolor A3(2). Nature 417, 141-147 (2002)

14. Ikeda, H. et al. Complete genome sequence and comparative analysis of the industrial microorganism Streptomyces avermitilis. Nat. Biotechnol. 21, 526-531 (2003)

15. van den Berg, M. A. et al. Genome sequencing and analysis of the filamentous fungus Penicillium chrysogenum. Nat. Biotechnol. 26, 1161-1168 (2008).

16. Bode, H. B., Bethe, B., Hofs, R. \& Zeeck, A. Big effects from small changes: possible ways to explore nature's chemical diversity. Chembiochem 3, 619-627 (2002).

17. Romano, S., Jackson, S. A., Patry, S. \& Dobson, A. D. W. Extending the "One strain many compounds"
(OSMAC) principle to marine microorganisms. Mar. Drugs 16, 244 (2018)

18. Wu, C. et al. Lugdunomycin, an angucycline-derived molecule with unprecedented chemical architecture Angew. Chem. Int. Ed. Engl. 58, 2809-2814 (2019).

19. Wright, E. S.\& Vetsigian, K. H. Inhibitory interactions promote frequent bistability among competing bacteria. Nat. Commun. 7, 11274 (2016).

20. Hibbing, M. E., Fuqua, C., Parsek, M. R. \& Peterson, S. B. Bacterial competition: surviving and thriving in the microbial jungle. Nat. Rev. Microbiol. 8 , 15-25 (2010)

21. Abrudan, M. I. et al. Socially mediated induction and suppression of antibiosis during bacterial coexistence. Proc. Natl Acad. Sci. USA 112, 11054-11059 (2015)

22. Traxler, M. F. \& Kolter, R. Natural products in soil microbe interactions and evolution. Nat. Prod. Rep. 32, 956-970 (2015)

23. Traxler, M. F., Watrous, J. D., Alexandrov, T., Dorrestein, P. C. \& Kolter, R. Interspecies interactions stimulate diversification of the Streptomyces coelicolor secreted metabolome. mBio 4, e00459-13 (2013).

This study uses nanospray desorption electrospray ionization mass spectrometry and MALDI-TOF imaging mass spectrometry to identify the chemical interactions that take place between actinomycete bacteria, revealing substantial changes in specialized metabolite production.

24. Rutledge, P. J. \& Challis, G. L. Discovery of microbial natural products by activation of silent biosynthetic gene clusters. Nat. Rev. Microbiol. 13, 509-523 (2015).

25. Zhu, H., Sandiford, S. K. \& van Wezel, G. P. Triggers and cues that activate antibiotic production by actinomycetes. J. Ind. Microbiol. Biotechnol. 41 , 371-386 (2014)

26. Guerinot, M. L. Microbial iron transport. Annu. Rev. Microbiol. 48, 743-772 (1994).

27. Li, C., Ji, C. \& Tang, B. Purification, characterisation and biological activity of melanin from Streptomyces sp. FEMS Microbiol. Lett. 365, fny077 (2018)

28. Sadeghi, A. et al. Diversity of the ectoines biosynthesis genes in the salt tolerant Streptomyces and evidence for inductive effect of ectoines on their accumulation. Microbiol. Res. 169, 699-708 (2014).

29. Kroiss, J. et al. Symbiotic streptomycetes provide antibiotic combination prophylaxis for wasp offspring. Nat. Chem. Biol. 6, 261-263 (2010).

30. Raaijmakers, J. M. \& Mazzola, M. Diversity and natural functions of antibiotics produced by beneficial and plant pathogenic bacteria. Annu. Rev. Phytopathol. 50, 403-424 (2012)

31. Doroghazi, J. R. \& Metcalf, W. W. Comparative genomics of actinomycetes with a focus on natural product biosynthetic genes. BMC Genomics 14, 61 (2013).

32. Cimermancic, P. et al. Insights into secondary metabolism from a global analysis of prokaryotic biosynthetic gene clusters. Cell 158, 412-421 (2014)

33. Sriswasdi, S., Yang, C. C. \& Iwasaki, W. Generalist species drive microbial dispersion and evolution. Nat. Commun. 8, 1162 (2017).

34. Shimkets, L. J. in Bacterial Genomes: Physical Structure and Analysis (eds de Bruijn, F. J., Lupski, J. R. \& Weinstock, G. M.) 5-11 (Chapman \& Hall, 1998)

35. Fraser, C. M. et al. The minimal gene complement of Mycoplasma genitalium. Science 270, 397-403 (1995).

36. Salem, H. et al. Drastic genome reduction in an herbivore's pectinolytic symbiont. Cell 171 . 1520-1531 (2017).

37. Davies, J. Millennium bugs. Trends Cell Biol. 9 M2-M5 (1999).

38. Claessen, D., Rozen, D. E., Kuipers, O. P., Sogaard-Andersen, L. \& van Wezel, G. P. Bacterial solutions to multicellularity: a tale of biofilms, filaments and fruiting bodies. Nat. Rev. Microbiol. 12 115-124 (2014)

39. Flärdh, K. \& Buttner, M. J. Streptomyces morphogenetics: dissecting differentiation in a filamentous bacterium. Nat. Rev. Microbiol. 7, 36-49 (2009).

40. Chater, K. F. \& Losick, R. in Bacteria as Multicellular Organisms (eds Shapiro, J. A. \& Dworkin, M.) 149-182 (Oxford University Press, 1997).

41. Merrick, M. J. A morphological and genetic mapping study of bald colony mutants of Streptomyces coelicolor. J. Gen. Microbiol. 96, 299-315 (1976)

42. Hopwood, D. A., Wildermuth, H. \& Palmer, H. M. Mutants of Streptomyces coelicolor defective in sporulation. J. Gen. Microbiol. 61, 397-408 (1970).

43. Bibb, M. J. Regulation of secondary metabolism in streptomycetes. Curr. Opin. Microbiol. 8, 208-215 (2005).

44. van der Heul, H. U., Bilyk, B. L., McDowall, K. J., Seipke, R. F. \& van Wezel, G. P. Regulation of antibiotic production in Actinobacteria: new perspectives from the post-genomic era. Nat. Prod. Rep. 35, 575-604 (2018).

45. Manteca, A. A death round affecting a young compartmentalized mycelium precedes aerial mycelium dismantling in confluent surface cultures of 
Streptomyces antibioticus. Microbiology 151, 3689-3697 (2005)

46. Manteca, A., Mader, U., Connolly, B. A. \& Sanchez, J. A proteomic analysis of Streptomyces coelicolor programmed cell death. Proteomics 6, 6008-6022 (2006).

47. Tenconi, E., Traxler, M. F., Hoebreck, C., van Wezel, G. P. $\&$ Rigali, S. Production of prodiginines is part of a programmed cell death process in Streptomyces coelicolor. Front. Microbiol. 9, 1742 (2018).

48. Ohnishi, Y. et al. Genome sequence of the streptomycinproducing microorganism Streptomyces griseus IFO 13350. J. Bacteriol. 190, 4050-4060 (2008).

49. Gomez-Escribano, J. P. et al. Structure and biosynthesis of the unusual polyketide alkaloid coelimycin P1, a metabolic product of the $c p k$ gene cluster of Streptomyces coelicolor M145. Chem. Sci. 3 2716-2720 (2012)

50. Wu, C. et al. Expanding the chemical space for natural products by Aspergillus-Streptomyces co-cultivation and biotransformation. Sci. Rep. 5, 10868 (2015)

51. Challis, G. L. ¿ Hopwood, D. A. Synergy and contingency as driving forces for the evolution of multiple secondary metabolite production by Streptomyces species. Proc. Natl Acad. Sci. USA 100, 14555-14561 (2003).

52. Ling, L. L. et al. A new antibiotic kills pathogens without detectable resistance. Nature 517, 455-459 (2015).

53. Wilson, M. C. et al. An environmental bacterial taxon with a large and distinct metabolic repertoire. Nature 506, 58-62 (2014).

54. Seipke, R. F., Kaltenpoth, M. \& Hutchings, M. I. Streptomyces as symbionts: an emerging and widespread theme? FEMS. Microbiol. Rev. 36 , 862-876 (2012).

55. Jensen, P. R., Williams, P. G., Oh, D.-C., Zeigler, L. $\&$ Fenical, W. Species-specific secondary metabolite production in marine actinomycetes of the genus Salinispora. Appl. Env. Microbiol. 73, 1146-1152 (2007).

56. Yang, A. et al. Nitrosporeusines A and B, unprecedented thioester-bearing alkaloids from the Arctic Streptomyces nitrosporeus. Org. Lett. 15, 5366-5369 (2013).

57. Sayed, A. M. et al. Extreme environments: microbiology leading to specialized metabolites. J. Appl. Microbiol. 128, 630-657 (2019)

58. Chevrette, M. G. et al. The antimicrobial potential of Streptomyces from insect microbiomes. Nat. Commun 10, 516 (2019).

This article describes the biosynthetic potential of insect-associated streptomycetes and the use of metabolomics for strain prioritization, resulting in the discovery of a novel antifungal agent active against multidrug-resistant fungal strains.

59. Zipperer, A. et al. Human commensals producing a novel antibiotic impair pathogen colonization. Nature 535, 511-516 (2016)

60. Ziemert, N. et al. Diversity and evolution of secondary metabolism in the marine actinomycete genus Salinispora. Proc. Natl Acad. Sci. USA 111 E1130-E1139 (2014).

This work shows high pathway diversity in recently diverged Salinispora spp., providing evidence for evolutionary drivers that allow bacteria to maximize their capacity to produce diverse secondary metabolites.

61. Adamek, M. et al. Comparative genomics reveals phylogenetic distribution patterns of secondary metabolites in Amycolatopsis species. BMC Genomics 19, 426 (2018).

62. Andam, C. P., Choudoir, M. J., Vinh Nguyen, A. Sol Park, H. \& Buckley, D. H. Contributions of ancestral inter-species recombination to the genetic diversity of extant Streptomyces lineages. ISME J. 10 , 1731-1741 (2016)

63. Tidjani, A. et al. Massive gene flux drives genome diversity between sympatric Streptomyces conspecifics. mBio 10, e01533-19 (2019).

64. McDonald, B. R. \& Currie, C. R. Lateral gene transfer dynamics in the ancient bacterial genus Streptomyces. mBio 8, e00644-17 (2017).

65. Medema, M. H., Cimermancic, P., Sali, A., Takano, E. \& Fischbach, M. A. A systematic computational analysis of biosynthetic gene cluster evolution: lessons for engineering biosynthesis. PLoS Comp. Biol. 10 e 1004016 (2014).

66. Joynt, R. \& Seipke, R. F. A phylogenetic and evolutionary analysis of antimycin biosynthesis. Microbiology 164, 28-39 (2018).
67. Chevrette, M. G. et al. Taxonomic and metabolic incongruence in the ancient genus Streptomyces. Front. Microbiol. 10, 2170 (2019).

68. Navarro-Munoz, J. C. et al. A computational framework to explore large-scale biosynthetic diversity. Nat. Chem. Biol. 16, 60-68 (2020). This work describes two computational tools, BiG-SCAPE and CORASON, designed to analyse BGC diversity in large genomic data sets.

69. Bruns, H. et al. Function-related replacement of bacterial siderophore pathways. ISME J. 12 320-329 (2018)

This study follows the evolutionary history of two siderophore biosynthesis pathways and reveals that acquisition of one pathway through HGT correlates to the loss of the other pathway.

70. Jensen, P. R. Natural products and the gene cluster revolution. Trends Microbiol. 24, 968-977 (2016).

71. Chater, K. F. \& Chandra, G. The evolution of development in Streptomyces analysed by genome comparisons. FEMS Microbiol. Rev. 30, 651-672 (2006).

72. Ventura, M. et al. Genomics of Actinobacteria: tracing the evolutionary history of an ancient phylum. Microbiol. Mol. Biol. Rev. 71, 495-548 (2007).

73. Choulet, F. et al. Evolution of the terminal regions of the Streptomyces linear chromosome. Mol. Biol. Evol. 23, 2361-2369 (2006)

74. Bilyk, B., Horbal, L. \& Luzhetskyy, A. Chromosomal position effect influences the heterologous expression of genes and biosynthetic gene clusters in Streptomyces albus J1074. Microb. Cell Fact. 16, 5 (2017).

75. Letzel, A.-C. et al. Genomic insights into specialized metabolism in the marine actinomycete Salinispora Env. Microbiol. 19, 3660-3673 (2017).

76. Ghinet, M. G. et al. Uncovering the prevalence and diversity of integrating conjugative elements in Actinobacteria. PLoS One 6, e27846 (2011).

77. Kinashi, H., Shimaji, M. \& Sakai, A. Giant linear plasmids in Streptomyces which code for antibiotic biosynthesis genes. Nature 328, 454-456 (1987).

78. Medema, M. H. et al. The sequence of a $1.8-\mathrm{Mb}$ bacterial linear plasmid reveals a rich evolutionary reservoir of secondary metabolic pathways. Genome Biol. Evol. 2, 212-224 (2010).

79. Mochizuki, S. et al. The large linear plasmid pSLA2-L of Streptomyces rochei has an unusually condensed gene organization for secondary metabolism. Mol. Microbiol. 48, 1501-1510 (2003)

80. Hoskisson, P. A. \& Fernández-Martínez, L. T. Regulation of specialised metabolites in Actinobacteria - expanding the paradigms. Env. Microbiol. Rep. 10 231-238 (2018)

81. Huang, J. et al. Cross-regulation among disparate antibiotic biosynthetic pathways of Streptomyces coelicolor. Mol. Microbiol. 58, 1276-1287 (2005).

82. McLean, T. C., Hoskisson, P. A. \& Seipke, R. F. Coordinate regulation of antimycin and candicidin biosynthesis. mSphere 1, e00305-16 (2016)

83. Martinet, L. et al. A single biosynthetic gene cluster is responsible for the production of bagremycin antibiotics and ferroverdin iron chelators. $\mathrm{mBio} 10$ e01230-19 (2019).

84. Liu, G., Chater, K. F., Chandra, G., Niu, G. \& Tan, H. Molecular regulation of antibiotic biosynthesis in Streptomyces. Microbiol. Mol. Biol. Rev. 77, 112-143 (2013).

85. Wietzorrek, A. \& Bibb, M. A novel family of proteins that regulates antibiotic production in streptomycetes appears to contain an OmpR-like DNA-binding fold. Mol. Microbiol. 25, 1181-1184 (1997)

86. Autret, S., Nair, R. \& Errington, J. Genetic analysis of the chromosome segregation protein Spo0J of Bacillus subtilis: evidence for separate domains involved in DNA binding and interactions with So protein. Mol. Microbiol. 41, 743-755 (2001).

87. Gramajo, H. C., Takano, E. \& Bibb, M. J. Stationaryphase production of the antibiotic actinorhodin in Streptomyces coelicolor A3(2) is transcriptionally regulated. Mol. Microbiol. 7, 837-845 (1993).

88. Tomono, A., Tsai, Y., Yamazaki, H., Ohnishi, Y. \& Horinouchi, S. Transcriptional control by A-factor of $s t r R$, the pathway-specific transcriptional activator for streptomycin biosynthesis in Streptomyces griseus. J. Bacteriol. 187, 5595-5604 (2005).

89. Lawlor, E. J., Baylis, H. A. \& Chater, K. F. Pleiotropic morphological and antibiotic deficiencies result from mutations in a gene encoding a tRNA-like product in Streptomyces coelicolor A3(2). Genes Dev. 1, 1305-1310 (1987).

90. Fernandez-Moreno, M. A., Caballero, J. L., Hopwood, D. A. \& Malpartida, F. The act cluster contains regulatory and antibiotic export genes, direct targets for translational control by the bldA tRNA gene of Streptomyces. Cell 66, 769-780 (1991).

91. Takano, E. $\gamma$-Butyrolactones: Streptomyces signalling molecules regulating antibiotic production and differentiation. Curr. Opin. Microbiol. 9, 287-294 (2006).

92. Willey, J. M. \& Gaskell, A. A. Morphogenetic signaling molecules of the streptomycetes. Chem. Rev. 111 174-187 (2011)

93. Tahlan, K. et al. Initiation of actinorhodin export in Streptomyces coelicolor. Mol. Microbiol. 63 951-961 (2007)

94. Wang, L. et al. Autoregulation of antibiotic biosynthesis by binding of the end product to an atypical response regulator. Proc. Natl Acad. Sci. USA 106, 8617-8622 (2009).

95. Willems, A. R. et al. Crystal structures of the Streptomyces coelicolor TetR-like protein ActR alone and in complex with actinorhodin or the actinorhodin biosynthetic precursor (S)-DNPA. J. Mol. Biol. 376, 1377-1387 (2008).

96. Francis, I. M., Jourdan, S., Fanara, S., Loria, R. \& Rigali, S. The cellobiose sensor CebR is the gatekeeper of Streptomyces scabies pathogenicity. mBio 6 , e02018 (2015).

97. Rigali, S. et al. Feast or famine: the global regulator DasR links nutrient stress to antibiotic production by. Streptomyces. EMBO Rep. 9, 670-675 (2008). This work is the first to describe a complete signalling pathway from an extracellular nutrient (GIcNAc) to the control of antibiotic biosynthetic pathways.

98. Nazari, B. et al. Chitin-induced gene expression involved in secondary metabolic pathways in Streptomyces coelicolor A3(2) grown in soil. Appl. Env. Microbiol. 79, 707-713 (2012).

99. Craig, M. et al. Unsuspected control of siderophore production by $\mathrm{N}$-acetylglucosamine in streptomycetes Env. Microbiol. Rep. 4, 512-521 (2012).

100. Świątek-Połatyńska, M. A. et al. Genome-wide analysis of in vivo binding of the master regulator DasR in Streptomyces coelicolor identifies novel non-canonical targets. PLoS One 10, e0122479 (2015).

101. Urem, M., Swiatek-Polatynska, M. A., Rigali, S. $\&$ van Wezel, G. P. Intertwining nutrient-sensory networks and the control of antibiotic production in Streptomyces. Mol. Microbiol. 102, 183-195 (2016).

102. Bode, H. Br., Bethe, B., Höfs, R. \& Zeeck, A. Big effects from small changes: possible ways to explore nature's chemical diversity. ChemBioChem 3, 619-627 (2002).

103. Zhu, H. et al. Eliciting antibiotics active against the ESKAPE pathogens in a collection of actinomycetes isolated from mountain soils. Microbiology 160 , 1714-1725 (2014).

104. Hosaka, T. et al. Antibacterial discovery in actinomycetes strains with mutations in RNA polymerase or ribosomal protein S12. Nat. Biotechnol. 27, 462-464 (2009)

105. Tanaka, Y. et al. Antibiotic overproduction by $r p s L$ and $r s m G$ mutants of various actinomycetes. Appl. Env. Microbiol. 75, 4919-4922 (2009).

106. Bertrand, S. et al. Metabolite induction via microorganism co-culture: a potential way to enhance chemical diversity for drug discovery. Biotechnol. Adv. 32, 1180-1204 (2014)

107. Hoshino, S., Wakimoto, T., Onaka, H. \& Abe, I. Chojalactones A-C, cytotoxic butanolides isolated from Streptomyces sp. cultivated with mycolic acid containing bacterium. Org. Lett. 17, 1501-1504 (2015).

108. Sugiyama, R. et al. 5-Alkyl-1,2,3,4-tetrahydroquinolines, new membrane-interacting lipophilic metabolites produced by combined culture of Streptomyces nigrescens and Tsukamurella pulmonis. Org. Lett. 17 1918-1921 (2015)

109. Hsiao, N. H., Gottelt, M. \& Takano, E. Chapter 6. Regulation of antibiotic production by bacterial hormones. Methods Enzymol. 458, 143-157 (2009).

110. Albright, J. C. et al. Large-scale metabolomics reveals a complex response of Aspergillus nidulans to epigenetic perturbation. ACS Chem. Biol., 1535-1541 (2015).

111. Craney, A., Ozimok, C., Pimentel-Elardo, S. M., Capretta, A. \& Nodwell, J. R. Chemical perturbation of secondary metabolism demonstrates important links to primary metabolism. Chem. Biol. 19, 1020-1027 (2012).

This work describes how screening a compound library for elicitors identified a novel family of molecules that activate antibiotic production

112. Moon, K., Xu, F. \& Seyedsayamdost, M. R. Cebulantin, a cryptic lanthipeptide antibiotic uncovered using 
bioactivity-coupled HiTES. Angew. Chem. Int. Ed. Engl. 58, 5973-5977 (2019).

113. Moon, K., Xu, F., Zhang, C. \& Seyedsayamdost, M. R. Bioactivity-HiTES unveils cryptic antibiotics encoded in actinomycete bacteria. ACS Chem. Biol. 14, 767-774 (2019)

114. Kramer, J., Ozkaya, O. \& Kummerli, R. Bacterial siderophores in community and host interactions. Nat. Rev. Microbiol. 18, 152-163 (2020)

115. Niu, G., Chater, K. F., Tian, Y., Zhang, J. \& Tan, H. Specialised metabolites regulating antibiotic biosynthesis in Streptomyces spp. FEMS Microbiol. Rev. 40, 554-573 (2016)

116. Onaka, H., Mori, Y., Igarashi, Y. \& Furumai, T. Mycolic acid-containing bacteria induce natural-product biosynthesis in Streptomyces species. Appl. Env. Microbiol 77 400-406 (2011).

117. Schroeckh, V. et al. Intimate bacterial-fungal interaction triggers biosynthesis of archetypa polyketides in Aspergillus nidulans. Proc. Natl Acad. Sci. USA 106, 14558-14563 (2009).

118. Sung A A Gromek, S. M \& Balunas, M. J. Upregulation and identification of antibiotic activity of a marine-derived Streptomyces sp. via co-cultures with human pathogens. Mar. Drugs 15 (2017).

119. Pérez, J. et al. Myxococcus xanthus induces actinorhodin overproduction and aerial mycelium formation by Streptomyces coelicolor. Microb. Biotechnol. 4, 175-183 (2011)

120. Patin, N. V., Floros, D. J., Hughes, C. C. Dorrestein, P. C. \& Jensen, P. R. The role of inter-species interactions in Salinispora specialized metabolism. Microbiology 164, 946-955 (2018).

121. Ezaki, M. et al. Biphenomycin A production by a mixed culture. Appl. Environ. Microbiol. 58, 3879-3882 (1992).

122. Kurosawa, K. et al. Rhodostreptomycins, antibiotics biosynthesized following horizontal gene transfer from Streptomyces padanus to Rhodococcus fascians. J. Am. Chem. Soc. 130, 1126-1127 (2008).

123. Traxler, M. F., Seyedsayamdost, M. R., Clardy, J. \& Kolter, R. Interspecies modulation of bacterial development through iron competition and siderophore piracy. Mol. Microbiol. 86, 628-644 (2012).

124. Onaka, H., Tabata, H., Igarashi, Y., Sato, Y. \& Furumai, T. Goadsporin, a chemical substance which promotes secondary metabolism and morphogenesis in streptomycetes. I. Purification and characterization J. Antibiot. 54, 1036-1044 (2001).

125. Yang, Y. L., Xu, Y., Straight, P. \& Dorrestein, P. C. Translating metabolic exchange with imaging mass spectrometry. Nat. Chem. Biol. 5, 885-887 (2009).

126. Mendes, R. et al. Deciphering the rhizosphere microbiome for disease-suppressive bacteria. Science 332, 1097-1100 (2011).

127. Currie, C. R., Scott, J. A., Summerbell, R. C. $\&$ Malloch, D. D. Fungus-growing ants use antibioticproducing bacteria to control garden parasites. Nature 398, 701-704 (1999)

128. Heine, D. et al. Chemical warfare between leafcutter an symbionts and a co-evolved pathogen. Nat. Commun. 9, 2208 (2018)

This work describes how Pseudonocardia, which protects a fungal garden against infection by a pathogenic fungus, elicits the production of counteracting antibiotics by the pathogen

129. Spaepen, S. in Principles of Plant-Microbe Interactions (ed. Lugtenberg, B.) 247-256 (Springer, 2015)

30. van der Meij, A. et al. Inter- and intracellular colonization of Arabidopsis roots by endophytic Actinobacteria and the impact of plant hormones on their antimicrobial activity. Antonie Van. Leeuwenhoek $111,679-690$ (2018) This study describes the interaction between plants and streptomycetes, and the chemical interaction through which plants might influence specialized metabolite production by endophytic Actinobacteria.

131. Blin, K. et al. antiSMASH 5.0: updates to the secondary metabolite genome mining pipeline. Nucleic Acids Res. 47, W81-W87 (2019).

This work describes a computational tool used by researchers worldwide to predict biosynthetic gene clusters from genome sequences.
132. Skinnider, M. A., Merwin, N. J., Johnston, C. W. \& Magarvey, N. A. PRISM 3. expanded prediction of natural product chemical structures from microbial genomes. Nucleic Acids Res. 45, W49-W54 (2017). PRISM3 predicts various potential chemical products of biosynthetic gene clusters, demonstrating how the field is moving towards natural product structure prediction from a genomic sequence.

133. Kautsar, S. A. et al. MIBiG 2.0: a repository for biosynthetic gene clusters of known function. Nucleic Acids Res. 48, D454-D458 (2019).

134. Doroghazi, J. R. et al. A roadmap for natural product discovery based on large-scale genomics and metabolomics. Nat. Chem. Biol. 10, 963-968 (2014). This work shows how GCF classification can be used to mine large data sets for biosynthetic potential.

135. Alanjary, M. et al. The Antibiotic Resistant Target Seeker (ARTS), an exploration engine for antibiotic cluster prioritization and novel drug target discovery. Nucleic Acids Res. 45, W42-W48 (2017).

136. Schorn, M. A et al Sequencing rare marine actinomycete genomes reveals high density of unique natural product biosynthetic gene clusters. Microbiology 162, 2075-2086 (2016).

137. Charlop-Powers, Z. et al. Global biogeographic sampling of bacterial secondary metabolism. eLife 4, e05048 (2015)

138. Charlop-Powers, Z. et al. Urban park soil microbiome are a rich reservoir of natural product biosynthetic diversity. Proc. Natl Acad. Sci. USA 113, 14811-14816 (2016).

139. Tiwari, K. \& Gupta, R. K. Rare actinomycetes: a potential storehouse for novel antibiotics. Crit. Rev. Biotechnol. 32, 108-132 (2012)

140. Donia, M. S. et al. A systematic analysis of biosynthetic gene clusters in the human microbiome reveals a common family of antibiotics. Cell $\mathbf{1 5 8 ,}$ 1402-1414 (2014)

141. Culp, E. J. et al. Hidden antibiotics in actinomycetes can be identified by inactivation of gene clusters for common antibiotics. Nat Biotechnol 37, 1149-1154 (2019).

This work shows how genome engineering of Actinobacteria by removing genes for common antibiotics may result in the production of novel metabolites that previously remained unidentified.

142. Hiard, S. et al. PREDetector: a new tool to identify regulatory elements in bacterial genomes. Biochem. Biophys. Res. Commun. 357, 861-864 (2007)

143. Tan, K., Moreno-Hagelsieb, G., Collado-Vides, J. \& Stormo, G. D. A comparative genomics approach to prediction of new members of regulons. Genome Res. 11, 566-584 (2001)

144. Rigali, S., Anderssen, S., Naome, A. \& van Wezel, G. P. Cracking the regulatory code of biosynthetic gene clusters as a strategy for natural product discovery. Biochem. Pharmacol. 153, 24-34 (2018).

145. Carrion, V. J. et al. Pathogen-induced activation of disease-suppressive functions in the endophytic root microbiome. Science 366, 606-612 (2019).

146. Duhrkop, K., Shen, H., Meusel, M., Rousu, J. ¿ Bocker, S. Searching molecular structure databases with tandem mass spectra using CSI:FingerID. Proc. NatI Acad. Sci. USA 112 12580-12585 (2015)

147. van der Hooft, J. J., Wandy, J., Barrett, M. P. Burgess, K. E. \& Rogers, S. Topic modeling fo untargeted substructure exploration in metabolomics. Proc. Natl Acad. Sci. USA 113, 13738-13743 (2016).

148. Mohimani, H. et al. Dereplication of peptidic natura products through database search of mass spectra. Nat. Chem. Biol. 13, 30-37 (2017).

149. Mohimani, H. et al. Dereplication of microbial metabolites through database search of mass spectra. Nat. Commun. 9, 4035 (2018)

150. Ernst, M. et al. MolNetEnhancer. enhanced molecular networks by integrating metabolome mining and annotation tools. Metabolites 9, 144 (2019).

151. Wilson, M. C. \& Piel, J. Metagenomic approaches for exploiting uncultivated bacteria as a resource for nove biosynthetic enzymology. Chem. Biol. 20, 636-647 (2013).
152. Smanski, M. J. et al. Synthetic biology to access and expand nature's chemical diversity. Nat. Rev. Microbiol 14, 135-149 (2016).

153. Sugimoto, Y. et al. A metagenomic strategy for harnessing the chemical repertoire of the human microbiome. Science 366, eaax9176 (2019). This paper describes an innovative strategy to identify and characterize biosynthetic gene clusters from uncultivated microbes using metagenomics.

154. Smanski, M. J. et al. Functional optimization of gene clusters by combinatorial design and assembly. Nat. Biotechnol. 32, 1241-1249 (2014).

155. Shomar, H. et al. Metabolic engineering of a carbapenem antibiotic synthesis pathway in Escherichia coli. Nat. Chem. Biol. 14, 794-800 (2018).

156. Amos, G. C. A. et al. Comparative transcriptomics as a guide to natural product discovery and biosynthetic gene cluster functionality. Proc. Natl Acad. Sci. USA 114, E11121-E11130 (2017).

157. Machado, H. Tuttle, R. N. \& Jensen, P. R. Omics-based natural product discovery and the lexicon of genome mining. Curr. Opin. Microbiol. 39, 136-142 (2017).

158. Badri, D. V. \& Vivanco, J. M. Regulation and function of root exudates. Plant. Cell Env. 32, 666-681 (2009).

159. Badri, D. V., Chaparro, J. M., Zhang, R., Shen, Q. \& Vivanco, J. M. Application of natural blends of phytochemicals derived from the root exudates of Arabidopsis to the soil reveal that phenolic-related compounds predominantly modulate the soil microbiome. J. Biol. Chem. 288, 4502-4512 (2013).

160. Bulgarelli, D. et al. Revealing structure and assembly cues for Arabidopsis root-inhabiting bacterial microbiota. Nature 488, 91-95 (2012).

161. Lebeis, S. L. et al. Salicylic acid modulates colonization of the root microbiome by specific bacterial taxa. Science 349, 860-864 (2015).

162. Culp, E. J. et al. Evolution-guided discovery of antibiotics that inhibit peptidoglycan remodelling. Nature 578, 582-587 (2020)

163. van Heel, A. J. et al. BAGEL4: a user-friendly web server to thoroughly mine RiPPs and bacteriocins. Nucleic Acids Res. 46, W278-W281 (2018).

164. Du, C. \& van Wezel, G. P. Mining for microbial gems: integrating proteomics in the postgenomic natural product discovery pipeline. Proteomics 18, e1700332 (2018).

165. Goering, A. W. et al. Metabologenomics: correlation of microbial gene clusters with metabolites drives discovery of a nonribosomal peptide with an unusual amino acid monomer. ACS Cent. Sci. 2, 99-108 (2016).

166. Soldatou, S., Eldjarn, G. H., Huerta-Uribe, A. Rogers, S. \& Duncan, K. R. Linking biosynthetic and chemical space to accelerate microbial secondary metabolite discovery. FEMS Microbiol. Lett. 366 fnz142 (2019)

167. Stokes, J. M et al. A deep learning approach to antibiotic discovery. Cell 180, 688-702 (2020).

Acknowledgements

This work is supported by NACTAR grant 16440 from the Netherlands Organization for Scientific Research (NWO) to M.H.M. and G.Pv.W., and by the Profile area 'Antibiotics' of the Faculty of Sciences of Leiden University.

Author contributions

The authors contributed equally to all aspects of the article.

Competing interests

M.H.M is a member of the Scientific Advisory Board of Hexagon Bio and co-founder of Design Pharmaceuticals. G.P.v.W. is in a committee for the Dutch Ministry of Health. D.A.v.B and B.R.T. do not declare any competing financial interests.

Publisher's note

Springer Nature remains neutral with regard to jurisdictional claims in published maps and institutional affiliations.

C) Springer Nature Limited 2020 